\title{
Induction of Haemeoxygenase-1 Improves FFA-Induced Endothelial Dysfunction in Rat Aorta
}

\author{
Fang Han ${ }^{a}$ Zongguang Hui ${ }^{b}$ Shuxian Zhang ${ }^{c}$ Ningning Hou ${ }^{b}$ Yali Wang ${ }^{b}$ \\ Xiaodong Sun ${ }^{b}$ \\ aDepartment of Pathology, Affiliated Hospital of Weifang Medical University, ${ }^{\text {bDepartment of }}$ \\ Endocrinology, Affiliated Hospital of Weifang Medical University, 'Department of Magnetic Resonance \\ Imaging, Medical Imaging Center, Affiliated Hospital of Weifang Medical University, Weifang, China
}

\section{Key Words}

Haemeoxygenase-1 $\bullet$ Free fatty acids • Endothelial dysfunction

\begin{abstract}
Background: The induction of haemeoxygenase-1 (HO-1) exerts beneficial effects in the setting of endothelial dysfunction in obesity. High free fatty acid (FFA) levels are a common feature of obesity and are the primary cause of endothelial dysfunction. The objective of our study was to explore the effects of HO-1 induction on FFA-induced endothelial dysfunction in rats. Methods: Rats received FFA treatment with either cobalt protoporphyrin (CoPP) to induce HO-1 or stannous protoporphyrin (SnPP) to inhibit HO-1. Endothelial function was determined by measuring endothelium-dependent vasodilatation (EDV). Nitric oxide (NO) production, superoxide production and nuclear factor (NF)-KB expression in the aorta were each determined. The levels of adenosine monophosphate (AMP)-activated kinase (AMPK) and endothelial nitric oxide synthase (eNOS) expression in endothelial cells were determined via Western blotting. Results: Induction of HO-1 by COPP decreased circulating FFA, highsensitivity $C$-reactive protein and malondialdehyde levels and increased serum adiponectin and glutathione levels compared with the FFA group $(P<0.05)$. High FFA levels resulted in EDV impairment, which was improved by HO- 1 induction $(P<0.05)$. Induction of $\mathrm{HO}-1$ increased NO levels and reduced aortic superoxide production and NF-KB expression compared with the FFA group. The FFA group exhibited decreased AMPK expression and eNOS phosphorylation, both of which were enhanced via HO-1 induction $(P<0.05)$. The beneficial effects of CoPP on EDV were partially attenuated in vitro in the presence of inhibitors of AMPK, phosphatidylinositol 3-kinase (PI3K), and eNOS. Conclusions: HO-1 induction with CoPP improves FFA-induced endothelial dysfunction in the rat aorta. The protective mechanism appears to be related to the activation of the AMPK-PI3K-eNOS pathway as a result of increased adiponectin levels as well as decreased inflammation and oxidative stress.
\end{abstract}

Copyright @ 2015 S. Karger AG, Basel

Xiaodong Sun

KARGER 125
Department of Endocrinology, Affiliated Hospital of Weifang Medical University, No.2428, Yuhe Road, Weifang, Shandong, 261031 (China)

Tel. +86-18653625906, E-Mail sxdfriend@sina.com 


\section{Introduction}

Many clinical observations and animal experiments have demonstrated that obesity is a major worldwide health care challenge $[1,2]$ and is an independent risk factor for ischemic cardiovascular disease [3]. Endothelial dysfunction is considered one of the primary causes of cardiovascular disease among obese people. In the setting of obesity, the release of excessive free fatty acids (FFA) from visceral fat results in endothelial lipotoxicity via increased levels of intracellular fatty acid metabolites and decreased mitochondrial fatty acid oxidation [4]. Increased inflammation and oxidative stress also alter endothelial nitric oxide synthase (eNOS) expression and activity, resulting in reduced nitric oxide (NO) production and low NO bioavailability, both of which regulate endothelial function.

The haeme oxygenase (HO) system is the primary pathway for haeme catabolism, which drives the conversion of haeme, a potentially harmful pro-oxidant, into biliverdin, iron, and carbon monoxide [5]. There are two HO isoenzymes: HO-1 and HO-2, the latter of which is constitutively expressed and accounts for the majority of $\mathrm{HO}$ activity. HO- 1 is inducible and is upregulated in response to both hypoxia and oxidative stress, exerting cytoprotective effects against oxidative insults [6].

Previous studies have shown that upregulation of HO-1 increases adiponectin levels by remodelling adipose tissue in both obese and diabetic rats [7, 8]. This relationship between HO-1 induction and increased adiponectin levels has resulted in the proposal of the existence of an HO-1/adiponectin axis [9-11]. Adiponectin is a cytokine secreted from adipose tissue that has both anti-atherogenic and insulin-sensitising properties [12]. Yu et al. [13] demonstrated that adiponectin improves endothelial function by increasing NO production and activating the adenosine monophosphate (AMP)-activated kinase (AMPK)eNOS pathway. These events decrease NO inactivation by blocking superoxide production in obese rats.

HO-1 induction has also recently been found to exert beneficial effects on cardiovascular health by normalising vascular function. Cao et al. [14] demonstrated that induction of HO-1 in association with increased levels of adiponectin prevents endothelial dysfunction in obese rats by blunting oxidative stress. Induction of HO-1 also attenuates weight gain and decreases inflammatory factors in both obese rats and obese diabetic mice, exerting beneficial effects on vascular function [15]. Given that elevated FFA levels are a common feature of obesity and are a primary cause of induced endothelial dysfunction, it is somewhat surprising that we still do not know whether HO-1 exerts a direct vascular protective effect on FFA-induced endothelial function. Therefore, the objectives of this study were to explore the potential effects of HO-1 induction on FFA-induced endothelial dysfunction in rats to better understand the mechanisms underlying these processes. We tested our hypothesis using an established method that entails infusing 20\% Intralipid and heparin to elevate plasma FFA levels [16] and cobalt protoporphyrin (CoPP) to induce HO-1. To verify that the effects of CoPP were due to increased HO-1 activity, we also concurrently treated the FFA group with stannous protoporphyrin (SnPP) to inhibit HO-1 activity.

\section{Materials and Methods}

\section{Materials}

Norepinephrine (NE), acetylcholine (ACh), and sodium nitroprusside (SNP) were each purchased from Sigma (St. Louis, MO, USA). Antibodies for the Western blots were obtained from Santa Cruz Biotechnology (Santa Cruz, California, USA). Adiponectin and high-sensitivity C-reactive protein (hs-CRP) assay kits were purchased from USCN Life Science Inc. (Wuhan, China). NO and malondialdehyde (MDA) assay kits were purchased from Beyotime Biotechnology (Beijing, China). CoPP and SnPP were purchased from Phoenix Pharmaceuticals, Inc., Burlingame, CA, US.

Experimental animals

Male Wistar rats (eight weeks-old) were purchased from Shandong Lukang Pharmaceutical Limited Company (Jining, China); their use was approved by the Institutional Animal Care and Use Committee 
(IACUC). The study conformed to the Guide for Care and Use of Laboratory Animals as published within Belgian Regulations. All of the rats were housed under standard laboratory conditions with free access to water and food. The rats were catheterised under sodium pentobarbital anaesthesia via a cannula inserted into the left carotid artery and silastic tubing inserted into the right jugular vein. All of the catheters were tunnelled under the skin and exteriorised on the upper back, and the rats were allowed to recover for 3 days before the experiments commenced. The rats were examined while awake and unstressed at $14 \mathrm{~h}$ following food withdrawal and then randomly divided into the following four groups: a normal control (NC) group, an FFA group, a CoPP group, and an SnPP group. The FFA group was infused for $6 \mathrm{~h}$ with $20 \%$ Intralipid $(18 \mu \mathrm{L} /$ $\mathrm{min})$ plus heparin (0.72 IU/min) to increase plasma FFA levels (FFA treatment) [17]. The CoPP group was also subjected to FFA treatment and simultaneously infused with CoPP ( $800 \mathrm{nmol} / \mathrm{kg} / \mathrm{min})$ [18] for $6 \mathrm{~h}$. The SnPP group was also subjected to FFA treatment and simultaneously infused with both CoPP $(800 \mathrm{nmol} /$ $\mathrm{kg} / \mathrm{min})$ and SnPP ( $800 \mathrm{nmol} / \mathrm{kg} / \mathrm{min})$ for $6 \mathrm{~h}$. The NC group was infused with normal saline alone $(18 \mu \mathrm{L} /$ min). To determine the effects of CoPP or SnPP on FFA treatment, the normal rats were also infused with CoPP or CoPP and SnPP as controls, respectively. Blood samples were collected at baseline and at the end of the infusions as described below. Following the infusions, intact thoracic aortas were immediately collected.

\section{Evaluation of endothelial function}

As has been described previously [19-21], intact thoracic aortas were immediately dissected and immersed in Krebs-Henseleit bicarbonate buffer ( $\mathrm{K}-\mathrm{H}$ solution; $95 \% \mathrm{O}_{2}$ and $5 \% \mathrm{CO}_{2}$ ) to measure endothelial function. The thoracic aortic vascular ring was then cut into 3-mm ring segments and immersed in individual organ chambers filled with $10 \mathrm{~mL}$ of $\mathrm{K}-\mathrm{H}$ solution at $37^{\circ} \mathrm{C}$. The rings were then mounted on two steel wires, as follows: one steel wire was fixed, and the other was inserted into the lumen and attached to the chamber and to an isometric force displacement transducer (PowerLab, AD Instruments, Dunedin, New Zealand) to record changes in force. The solution was continuously gassed with $95 \% \mathrm{O}_{2}$ and $5 \% \mathrm{CO}_{2}$ and maintained at $37^{\circ} \mathrm{C}$. The rings were equilibrated for $60 \mathrm{~min}$ with a resting tension of $1.0 \mathrm{~g}$. During this time, the tissues were washed every $30 \mathrm{~min}$ with $\mathrm{K}-\mathrm{H}$ solution. Each of the rings was first contracted with $\mathrm{KCl}(60 \mathrm{mM})$ and subsequently rinsed several times in K-H solution. Before the addition of ACh $\left(10^{-8}\right.$ to $\left.10^{-4} \mathrm{~mol} / \mathrm{L}\right)$ or SNP $\left(10^{-8}\right.$ to $\left.10^{-4} \mathrm{~mol} / \mathrm{L}\right)$ to the solution to assess the EDV or the endothelium-independent vasodilatation (EIV) response of the arterial rings, NE $(1 \mu \mathrm{M})$ was added to induce a steady contraction. The collected data were used to prepare concentration-relaxation response curves.

\section{Plasma measurements}

Plasma FFA, serum glutathione (GSH), and MDA concentrations were measured via a colorimetric assay according to the manufactures' instructions. Serum adiponectin and hs-CRP levels were determined using commercially available enzyme-linked immunosorbent assay (ELISA) kits.

\section{Determination of total NO production}

Following the determination of endothelial function, total NO production (nitrite and nitrate) by the aortic rings was determined via a modified Griess reaction method as described previously [22, 23]. Briefly, after the ACh $\left(10^{-4} \mathrm{M}\right)$ was added, $100 \mu \mathrm{L}$ of K-H solution was taken from the organ bath and mixed with an equal volume of modified Griess reagent. Following 10 min of incubation at room temperature, the NO concentration was spectrophotometrically determined at $540 \mathrm{~nm}$ according to the manufacturer's instructions. The values were estimated per the amount of dry weight in the tissue.

Measurement of HO activity in the aorta

HO activity was assayed in the aortic homogenates as described previously [24]. Briefly, spectrophotometry was used to measure the concentration of bilirubin, the end product of haeme degradation, using the difference in absorption between 464 and $530 \mathrm{~nm}$.

\section{Measurement of superoxide production in the aorta}

Superoxide production in the rat aorta was determined via the lucigenin chemiluminescence method as described previously $[25,26]$. Superoxide production was expressed as counts per minute per milligram of aortic tissue. 
Immunohistochemistry

Portions of the aorta were collected for NF-KB immunohistochemistry. Briefly, the aortic rings were fixed in $10 \%$ formalin, sectioned and then used for NF- $\mathrm{\kappa B}$ immunohistochemistry. After restoring antigen retrieval, the sections were incubated with an anti-NF-KB antibody (Beijing Zhongshan Biotechnology Co., Ltd., Beijing, China) and a secondary antibody. Sections were then counterstained with haematoxylin and examined via light microscopy. Six fields per animal (three samples $\times$ two fields) were analysed, and the numbers of NF- $\mathrm{kB}$ p65-positive nuclei and cells were calculated using Image-Pro plus 6.0 (Media Cybernetics, Rockville, MD, USA).

\section{Western blot analysis}

The extracted proteins from the aorta and the endothelial cells were subjected to sodium dodecyl sulphate-polyacrylamide gel electrophoresis (SDS-PAGE). The proteins were then transferred onto nitrocellulose membranes and blocked with a solution of Tris-buffered saline containing $5 \%$ fat-free milk and $0.1 \%$ Tween-20 (TBST) at room temperature for 1 hour, probed with different primary antibodies against AMPK, eNOS, p-eNOS (Ser 1177), HO-1 and HO-2, and washed three times in TBST. The membranes were incubated with secondary antibodies for 1 hour, washed with TBST and stained with nitro blue tetrazolium/5-bromo-4-chloro-3-indolyl phosphate. The protein expression levels were quantified using Image Pro-plus 6.0.

\section{Signalling pathway studies}

To investigate the potential mechanism by which CoPP affects FFA-induced endothelial dysfunction, the aortic rings obtained from the CoPP treated groups were incubated with the following specific inhibitors of the candidate pathways: (1) $20 \mu \mathrm{M}$ of the AMPK inhibitor compound C (Merck, Darmstadt, Germany); (2) $30 \mu \mathrm{M}$ of the PI3K inhibitor LY294002 (Sigma, St. Louis, MO, USA); and (3) $1 \mathrm{mM}$ of the eNOS inhibitor L-NAME (Sigma). Following 60 min of incubation, the aortic rings were pre-contracted with norepinephrine $(1 \mu \mathrm{M})$ followed by either ACh $\left(10^{-8}-10^{-4} \mathrm{M}\right)$ or SNP $\left(10^{-8}-10^{-4} \mathrm{M}\right)$ to determine the EDV and EIV.

\section{Statistical analysis}

All of the data were subjected to a one-way analysis of variance (ANOVA) statistical analysis using the SPSS 16.0 statistical package (IBM, Chicago, IL, USA), and the data were expressed as the mean \pm standard deviation (SD). The Student-Newman-Keuls method was used to detect significant differences among the group comparisons. A $P$ value of less than 0.05 was considered statistically significant.

\section{Results}

Biometric and blood parameters of rats in the study groups

We did not observe a significant difference between the FFA levels in the basal state (Table 1) $(P>0.05)$. However, the rats in the FFA group exhibited significantly increased FFA levels following the infusion of Intralipid and heparin compared with the NC group. In contrast, the CoPP group exhibited a reduction in FFA levels compared with the FFA group $(P<0.05)$. Additionally, the FFA group exhibited increased circulating hs-CRP and MDA levels as well as decreased serum adiponectin and GSH levels compared with the NC group $(P<0.05)$. Interestingly, the CoPP rats exhibited decreased circulating hs-CRP and MDA levels and increased serum adiponectin and GSH levels $(P<0.05)$. However, there were no significant differences in the above indexes between the FFA and SnPP-treated rats $(P>0.05)$. In the normal rats treated with either CoPP or SnPP, no differences in the above indexes were observed compared with the NC group.

\section{Endothelial function in rats}

ACh-induced EDV in the thoracic aorta was attenuated in the FFA group compared with the NC group. However, the attenuation decreased following CoPP treatment $(P<0.05)$; this effect was reversed via treatment with SnPP. Notably, no significant differences in SNPinduced EIV were noted among the four groups (Fig. 1). Additionally, in the normal rats 
Han et al.: HO-1 Improves Endothelil Function

Table 1. Blood parameters of Rats in the Studied Groups (n=7-8). Data are shown as mean \pm SD. FFAs, free fatty acids; Hs-CRP, high-sensitive $C$ reaction protein; MDA, malondialdehyde; GSH, glutathione; NC, normal control group; FFA, the group treated with FFA; CoPP, the group treated with FFA and CoPP; SnPP, the group treated with FFA, CoPP and SnPP; NC-CoPP, normal control group treated with CoPP; NC-SnPP, normal control group treated with CoPP and SnPP; ${ }^{*} \mathrm{P}<0.01$ vs NC group. ${ }^{\#} \mathrm{P}<0.01$ vs FFA group

\begin{tabular}{ccccccc}
\hline Group & $\begin{array}{c}\text { FFA(mmol/L) } \\
\text { (before infuse) }\end{array}$ & $\begin{array}{c}\text { FFA(mmol/L) } \\
\text { (after infuse) }\end{array}$ & $\begin{array}{c}\text { Adiponectin } \\
\text { (ug/L) }\end{array}$ & $\begin{array}{c}\text { Hs-CRP } \\
\text { (mg/L) }\end{array}$ & $\begin{array}{c}\text { MDA } \\
\text { (umol/L) }\end{array}$ & $\begin{array}{c}\text { GSH } \\
\text { (mg/L) }\end{array}$ \\
\hline NC & $0.33 \pm 0.11$ & $0.40 \pm 0.12$ & $210.4 \pm 30.6$ & $0.89 \pm 0.25$ & $2.45 \pm 0.54$ & $209.5 \pm 35.6$ \\
FFA & $0.34 \pm 0.13$ & $1.26 \pm 0.30^{*}$ & $88.6 \pm 27.0^{*}$ & $2.05 \pm 0.59^{*}$ & $5.87 \pm 0.71^{*}$ & $156.2 \pm 30.2^{*}$ \\
CoPP & $0.36 \pm 0.14$ & $0.65 \pm 0.31^{\#}$ & $190.3 \pm 32.6^{\#}$ & $1.08 \pm 0.45^{\#}$ & $2.39 \pm 0.51^{\#}$ & $220.8 \pm 40.4^{\#}$ \\
SnPP & $0.40 \pm 0.17$ & $1.15 \pm 0.41^{*}$ & $92.73 \pm 30.3^{*}$ & $2.19 \pm 0.48^{*}$ & $5.55 \pm 0.47^{*}$ & $163.5 \pm 28.4^{*}$ \\
NC-CoPP & $0.31 \pm 0.12$ & $0.45 \pm 0.10$ & $228.4 \pm 24.5$ & $0.90 \pm 0.21$ & $2.25 \pm 0.61$ & $190.5 \pm 40.1$ \\
NC-SnPP & $0.32 \pm 0.10$ & $0.43 \pm 0.14$ & $218.5 \pm 21.8$ & $0.95 \pm 0.27$ & $2.38 \pm 0.56$ & $201.7 \pm 38.9$ \\
\hline
\end{tabular}

Fig. 1. Vasorelaxation response of thoracic aorta segment to Ach (A) and SNP (B) in the studied groups. ACh-induced EDV in thoracic aorta was attenuated in the FFA group compared with the NC group. However, attenuation decreased following CoPP treatment and this effect was reversed by treatment with SnPP. Notably, no significant differences in SNP-induced EIV were found between the four groups. N= 7-8 vascular segments/group from 7-8 rats. Data are shown as mean $\pm \mathrm{SD}$. ${ }^{*} \mathrm{P}<0.05$ vs $\mathrm{NC}$ group. ${ }^{\#} \mathrm{P}<0.05$ vs FFA group.

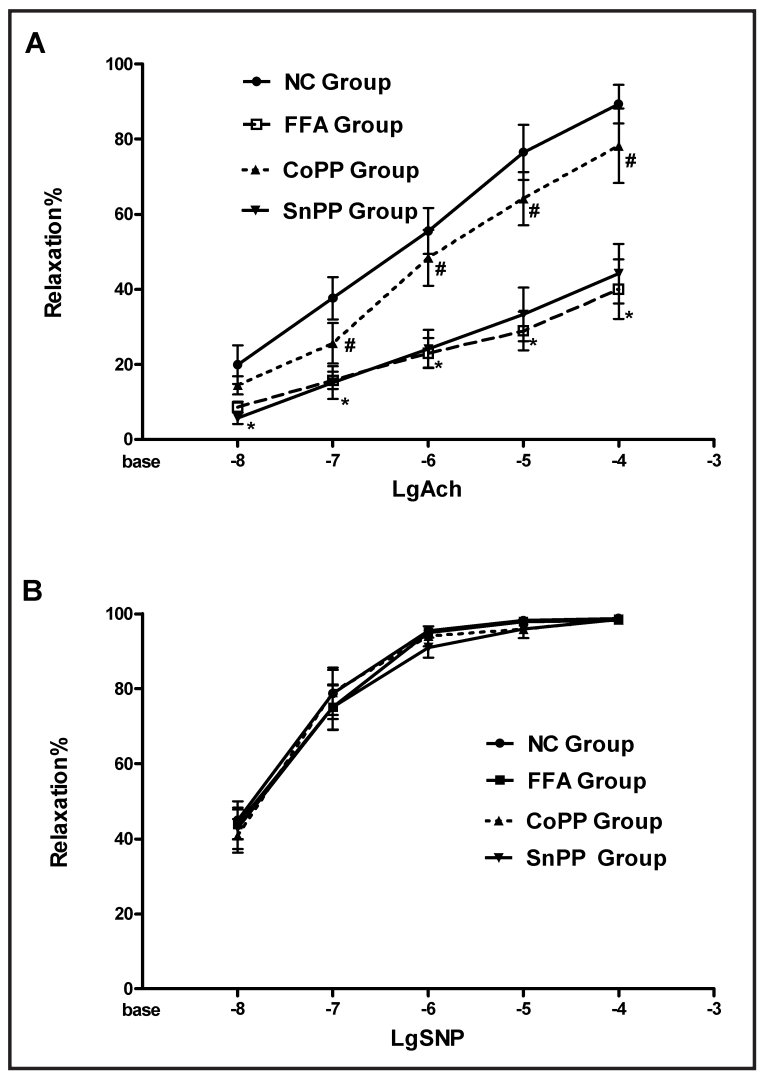

Fig. 2. Total NO production of the endothelium from the studies groups. NO concentration was significantly reduced in the FFA group compared with the controls. However, NO levels from the CoPP group were substantially increased compared with those from the FFA group. There was no difference between the FFA and SnPP groups. N = 7-8 vascular segments/group from 7-8 rats. Data are shown as mean \pm SD. ${ }^{*} \mathrm{P}<0.05$ vs NC group; ${ }^{*} \mathrm{P}<0.05$ vs FFA group.

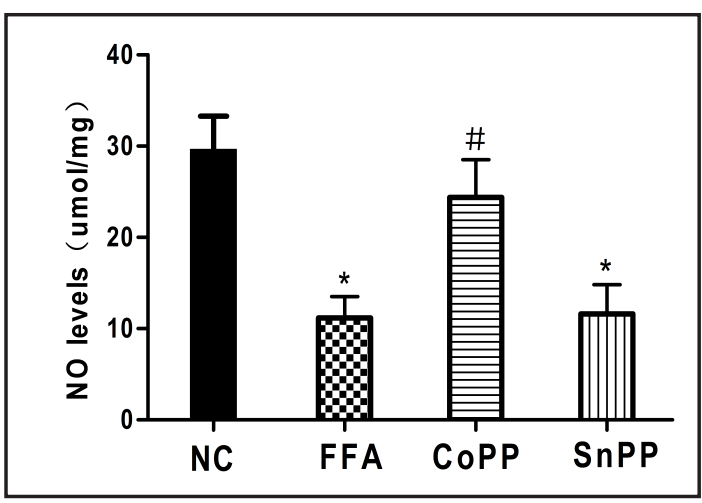

treated with either CoPP or SnPP, no differences in EDV were noted compared with the NC group $(P>0.05$, data not shown). 


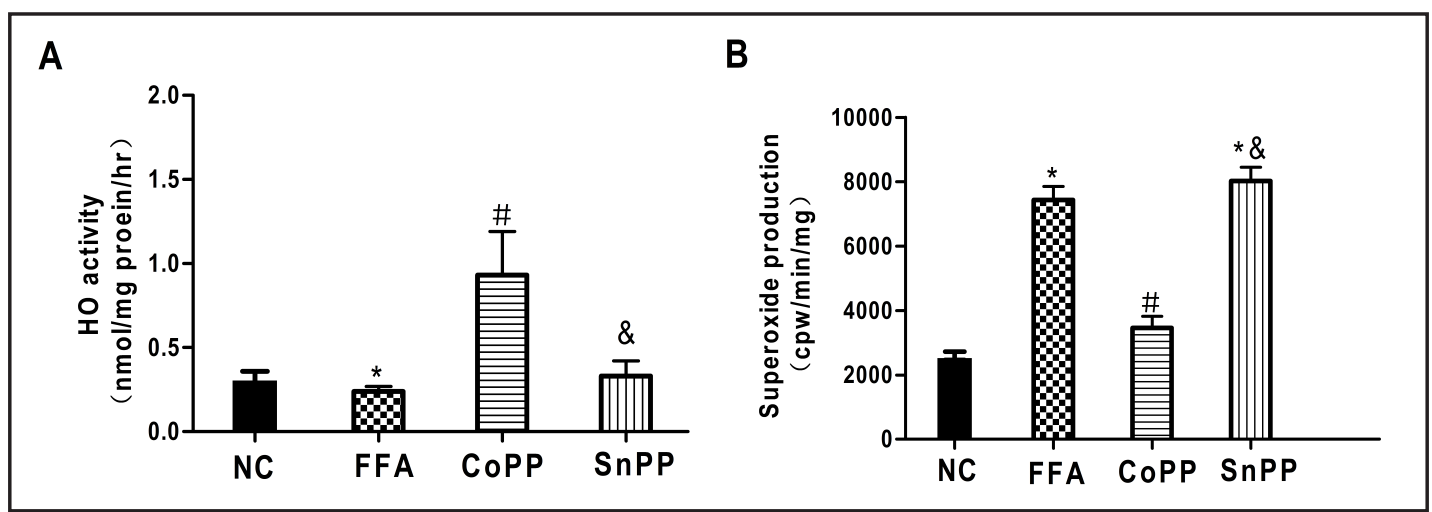

Fig. 3. HO activity (A) and superoxide production (B) of the aorta 'from the studies groups. HO activity was decreased and superoxide production was increased in the FFA group compared with the NC group. and CoPP increased HO activity and decreased superoxide production. N = 7-8 vascular segments/group from $7-8$ rats. Data are shown as mean $\pm \mathrm{SD}$. ${ }^{*} \mathrm{P}<0.05 \mathrm{vs}$ NC group; ${ }^{\#} \mathrm{P}<0.05$ vs FFA group; ${ }^{\&} \mathrm{P}<0.05$ vs CoPP group.

Fig. 4. $N F-\kappa B$ expression of vascular ring in the rats. Thoracic aortic endothelial cells showed increased NF- $\mathrm{B}$ expression in the FFA group compared with the NC group (positive cells, $82.6 \pm 7.4 \%$ vs. $18.5 \pm 2.1 \%$ ). However, CoPP treatment decreased NF$\kappa \mathrm{B}$ expression ( $43.6 \pm 8.3 \%$ vs. $82.6 \pm$ $7.4 \%$ ). No significant difference was found in SnPP and FFA group (80.1 \pm $9.2 \%$ vs. $82.6 \pm 7.4 \%$ ). A NC group; B FFA group; C CoPP group; D SnPP group.

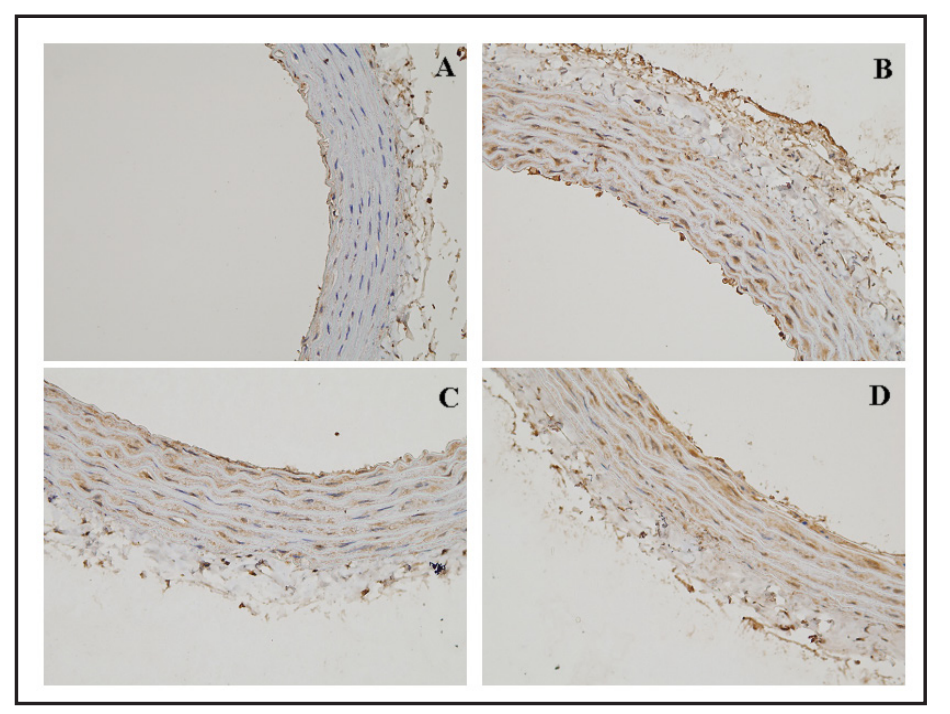

Total NO production in the aorta

To further explore the possibility that CoPP may increase NO production, we measured the NO levels in the K-H solutions of each of the four experimental groups. As shown in Figure 2, the NO concentration was significantly decreased in the FFA group compared with the NC group $(P<0.05)$. However, the NO levels in the CoPP group were significantly increased compared with the FFA group $(P<0.05)$. There was no difference between the FFA and SnPP groups $(P>0.05)$.

\section{HO activity in the aorta}

$\mathrm{HO}$ activity was measured in the aortas isolated from all four groups (Fig. 3A). We observed a significant decrease in $\mathrm{HO}$ activity in the FFA group compared with the NC group $(P<0.05)$; CoPP increased HO activity in the aorta, whereas SnPP abolished this increase $(P<0.05)$.

Superoxide production in the aorta

Aortic superoxide production levels were increased in the FFA group compared with the NC group $(P<0.05)$. In contrast, CoPP-treated rats exhibited decreased aortic superoxide 
Fig. 5. Western blot analysis for the expressions of the HO- 1 and HO-2 proteins in the aorta. *P $<0.05$ vs NC group; \#P $<0.05$ vs FFA group; ${ }^{\&} \mathrm{P}<0.05$ vs CoPP group.

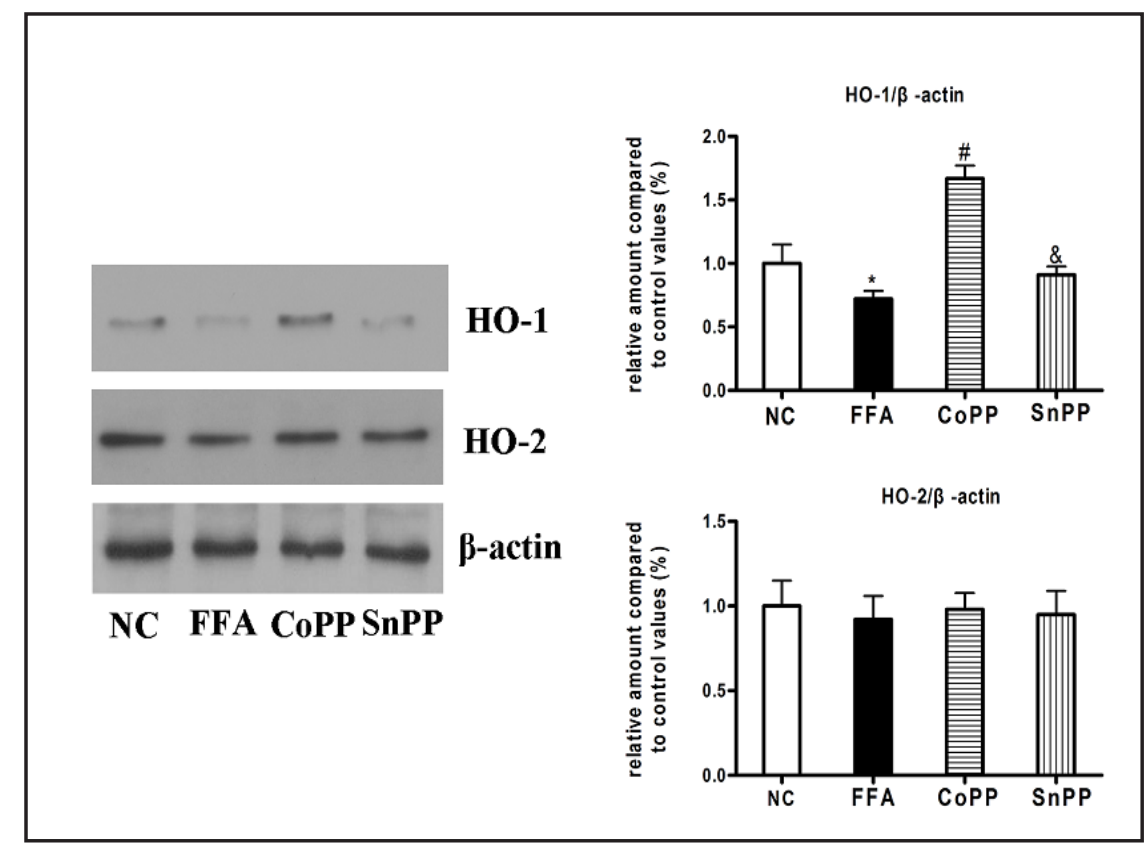

Fig. 6. Western blot analysis for the expressions of the AMPK and eNOS proteins in the endothelial cells. ${ }^{*} \mathrm{P}<$ 0.05 vs NC group; "P $<0.05$ vs FFA group; ${ }^{\&} \mathrm{P}<0.05$ vs CoPP

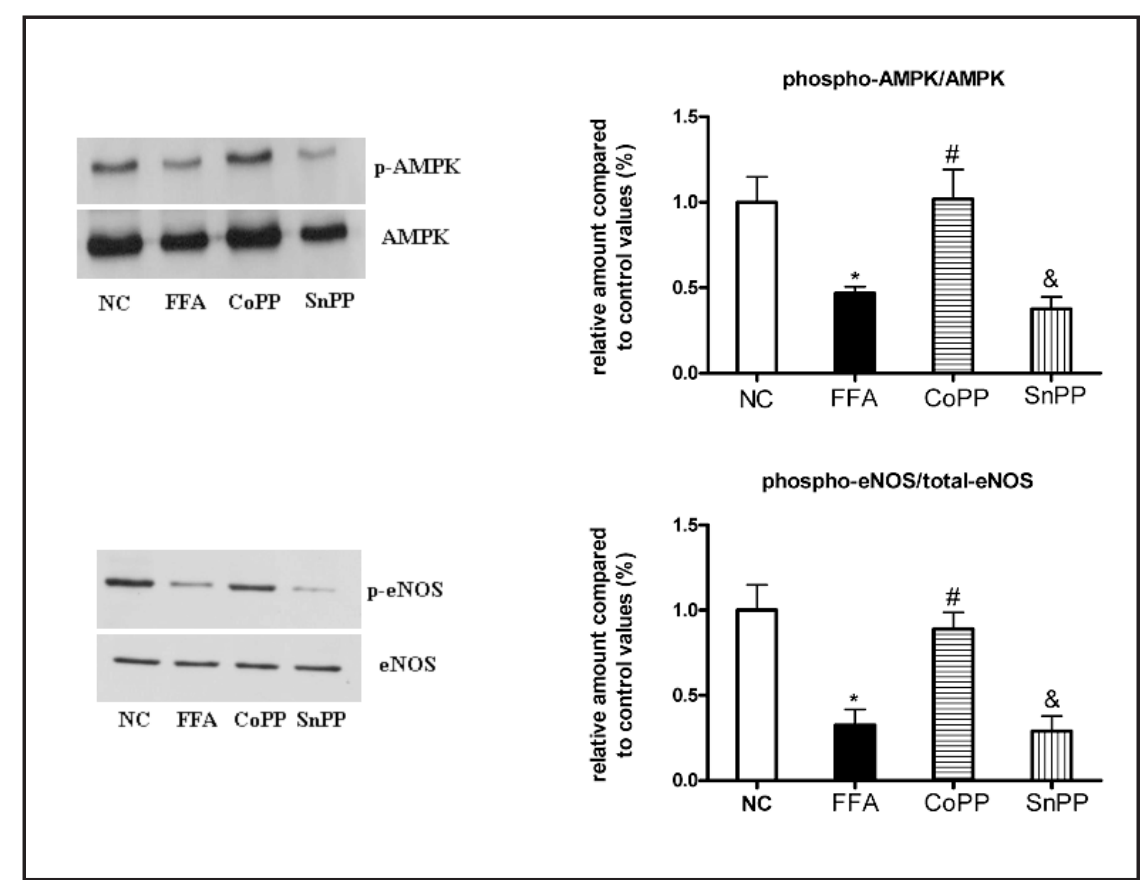
group.

production compared with the FFA group $(P<0.05)$. Interestingly, SnPP inhibited HO activity compared with the CoPP-treated rats, which exhibited increased superoxide production (Fig. 3B).

\section{Immunohistochemistry}

The thoracic aortic endothelial cells exhibited increased NF- $\kappa$ B expression in the FFA group compared with the $\mathrm{NC}$ group, as determined via immunohistochemistry (positive cells, $82.6 \pm 7.4 \%$ vs. $18.5 \pm 2.1 \%$ ). However, CoPP treatment decreased NF- $\kappa$ B expression $(43.6 \pm 8.3 \%$ vs. $82.6 \pm 7.4 \%)$. No significant difference was observed between the SnPP and FFA groups ( $80.1 \pm 9.2 \%$ vs. $82.6 \pm 7.4 \%$ ) (Fig. 4 ). 
Fig. 7. Vasorelaxation response of thoracic aorta segment to Ach in the studied groups. CoPP treatment improved ACh-induced EDV attenuation in the FFA group. However, the beneficial effect of CoPP was partly attenuated in the presence of AMPK inhibitor, PI3K inhibito, or NO synthase inhibitor. $\mathrm{N}=7-8$ vascular segments/group from 7-8 rats. Data are shown as mean \pm SD. $* \mathrm{P}<0.05$ vs NC group. ${ }^{\#} \mathrm{P}<0.05$ vs FFA group. ${ }^{\&} \mathrm{P}<0.05 v$ s CoPP group.

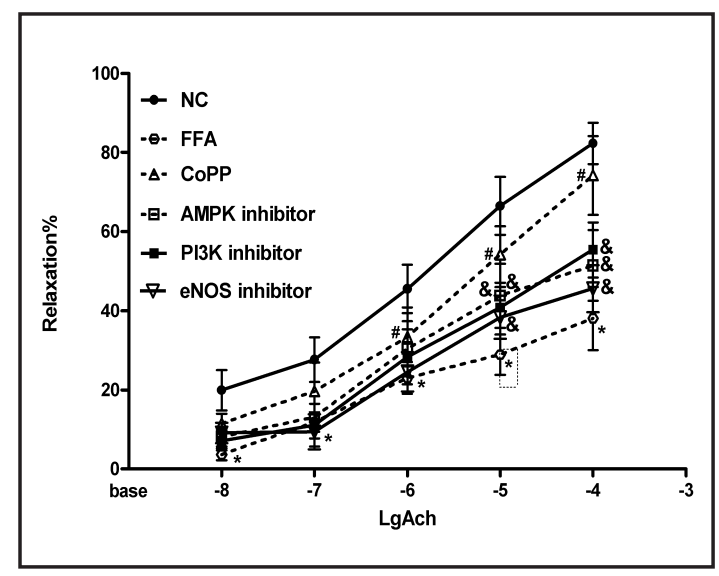

Western blotting for $\mathrm{HO}-1$ and $\mathrm{HO}-2$ levels in the aortas

The rats in the FFA group exhibited lower HO-1 protein levels compared with the NC group $(P<0.05$, Fig. 5). CoPP treatment caused an increase in the HO-1 protein levels in the aorta, whereas SnPP treatment abolished this increase $(P<0.05)$. There were no significant differences in the levels of HO-2 protein.

Western blotting for AMPK and eNOS expression in the endothelial cells

As shown in Figure 6, the FFA group exhibited decreased AMPK and eNOS phosphorylation $(P<0.05)$. However, CoPP treatment enhanced both AMPK and eNOS phosphorylation $(P<0.05)$.

\section{Signalling pathway studies}

As shown above, CoPP treatment improved ACh-induced EDV attenuation in the FFA group. However, the beneficial effect exerted by CoPP was partially attenuated in the presence of the AMPK inhibitor compound C, the PI3K inhibitor LY294002, and the NO synthase inhibitor L-NAME (Fig. 7). SNP-induced EIV changes were not statistically significant among the four groups (data not shown).

\section{Discussion}

In this study, we demonstrated that induction of HO-1 with CoPP improves FFA-induced endothelial dysfunction in rats. The mechanism for this protective effect is related to the activation of the AMPK-PI3K-eNOS pathway, which is induced by increasing adiponectin levels in parallel with reduced inflammation and oxidative stress.

The pathogenic relationship between obesity and cardiovascular disease is well established [27]. Endothelial dysfunction, a critical component of atherosclerosis development, plays a critical role in both the onset and the development of cardiovascular disease and is observed in obesity-associated metabolic syndrome [13]. Several risk independent factors for endothelial dysfunction have been identified in the setting of obesity, including elevated circulating FFA levels [13]. Elevated FFA levels impair endothelial cell NO production by inducing chronic inflammation and oxidative stress. In our study, the rats in the FFA group exhibited significantly increased FFA levels following the infusion of Intralipid and heparin, indicating the successful establishment of the high FFA model. As expected, the ACh-induced EDV in the aorta was attenuated in the FFA group compared with the NC group, a finding consistent with previous studies [17].

The HO system exerts its antioxidant properties via its by-products: biliverdin, iron, and carbon monoxide [5]. H0-1 is upregulated in response to oxidative stress and plays a crucial protective role against oxidative insult in obesity and diabetes-induced cardiovascular disease $[6,28]$. H0-1 induction prevents endothelial dysfunction by decreasing oxidant 
production [29]. Recent studies have demonstrated that HO-1 induction increases serum adiponectin levels by remodelling adipose tissue in obese and diabetic rats $[7,8,30]$, a relationship referred to as the HO-1/adiponectin axis [9-11]. In our study, the HO-1 activator CoPP was used to induce HO-1 activity, whereas SnPP was used to inhibit HO-1 activity. Our results indicated that CoPP-induced HO-1 increased both EDV and serum adiponectin levels in rats infused with Intralipid and heparin. However, inhibition of HO-1 with SnPP did not significantly increase either EDV or serum adiponectin levels. These results suggest that HO-1 induction improved FFA-induced endothelial dysfunction and that this beneficial effect is associated with increased adiponectin levels.

EDV in the aorta is related to endothelial-derived NO, which is induced via eNOS phosphorylation.Previousstudieshavedemonstrated thatFFA-inducedeNOSphosphorylation accounts for endothelial function in the setting of obesity [13]. We observed decreased eNOS phosphorylation and the NO concentration in the FFA group compared with the NC group. We also observed that CoPP-induced HO-1 increased eNOS phosphorylation and the NO concentration. These changes were not observed following SnPP-induced inhibition of HO-1 activity. In addition, the HO-1-induced improvement in endothelial function was abolished by the eNOS-specific inhibitor L-NAME in vitro. These results suggest that HO-1 induction protects against endothelial dysfunction by increasing NO levels via eNOS phosphorylation

As mentioned above, HO-1 induction increases circulating adiponectinlevels. Adiponectin is a cytokine secreted primarily by adipose tissue that exerts vasoprotective effects in the setting of cardiovascular disease. Some studies have shown that hypoadiponectinaemia is associated with endothelial dysfunction and that adiponectin-deficient mice exhibit impaired EDV and decreased NO production [31, 32]. Furthermore, adiponectin may independently protect against the endothelial dysfunction caused by high concentrations of FFA via the activation of the AMPK/PI3K/eNOS pathway and NO production $[13,33]$. We observed that CoPP treatment enhanced both AMPK and eNOS phosphorylation and improved endothelial function, although these effects were abolished following treatment with inhibitors of AMPK, $\mathrm{PI} 3 \mathrm{~K}$, and eNOS. These results indicate that HO-1 induction may partially improve endothelial function via the AMPK/PI3K/eNOS pathway. Additionally, FFA-induced inflammation and oxidative stress also cause endothelial dysfunction. Low adiponectin levels appear to be linked to both increased inflammation and oxidative stress [34]. In addition, induction of HO- 1 by CoPP increases adiponectin and GSH levels but decreases hs-CRP and MDA levels as well as aortic superoxide anion production and NF- $\mathrm{BB}$ expression in rats treated with FFA, whereas SnPP-induced inhibition of HO-1 activity does not. These results suggest that HO-1 induction may improve endothelial function via increased adiponectin levels and decreased inflammation and oxidative stress. Thus, HO-1 appears to play a critical role in cellular defence against FFA-induced endothelial dysfunction.

It is interesting that we observed that induction of HO- 1 with CoPP reduced FFA levels. Adiponectin reduces plasma FFA levels by enhancing the $\beta$-oxidation of FFA in muscle [35]. Therefore, inducing HO-1 may reduce circulating FFA levels by increasing adiponectin levels via the HO-1/adiponectin axis $[9,10]$. In addition, our conclusion that induction of HO-1 may improve FFA-induced endothelial dysfunction is based upon studies in which we examined the acute effects of induction of HO-1 on endothelial function. This conclusion contrasts with previous studies in which transgenic mice overexpressing either HO-1 or CoPP were subjected to longer periods of HO-1 induction; these studies demonstrated that the induction of HO- 1 did not improve endothelial function [36, 37]. It is possible that the acute induction of HO-1 in these vessels may have had a different effect on vascular relaxation compared with chronic induction of HO-1. A possible explanation for this finding may be related to the various actions of the by-products of $\mathrm{HO}-1$ induction, including $\mathrm{CO}$, on vascular function under different conditions. However, the specific molecular mechanism underlying the phenomenon mentioned above must be clarified in future studies.

In conclusion, we have demonstrated that CoPP-induced HO-1 improves FFA-induced endothelial dysfunction in rats. The mechanism underlying this protective effect is associated with the activation of the AMPK-PI3K-eNOS pathway as well as increased adiponectin

\section{KARGER}


levels and reduced inflammation and oxidative stress. In summary, these findings may have important clinical implications in the management and treatment of cardiovascular disease in obese patients.

\section{Acknowledgements}

We would like to thank the American Journal Experts for providing language editing assistance for our manuscript. This work was supported by the National Natural Science Foundation of China (Grants NO. 81300688 and 81400829), and the Science and Technology Innovation Fund of Weifang Medical University (NO. K1301021, NO. K1302017).

\section{Disclosure Statement}

The authors declare that they have no conflict of interest

\section{References}

1 Hurt RT, Frazier TH, McClave SA, Kaplan LM: Obesity epidemic: overview, pathophysiology, and the intensive care unit conundrum. JPEN J Parenter Enteral Nutr 2011;35:4S-13S.

2 Rokholm B, Baker JL, Sorensen TI: The levelling off of the obesity epidemic since the year 1999--a review of evidence and perspectives. Obes Rev 2010;11:835-846.

- 3 Poirier P, Giles TD, Bray GA, Hong Y, Stern JS, Pi-Sunyer FX, Eckel RH: Obesity and cardiovascular disease: pathophysiology, evaluation, and effect of weight loss: an update of the 1997 American Heart Association Scientific Statement on Obesity and Heart Disease from the Obesity Committee of the Council on Nutrition, Physical Activity, and Metabolism. Circulation 2006;113:898-918.

4 Chinen I, Shimabukuro M, Yamakawa K, Higa N, Matsuzaki T, Noguchi K, Ueda S, Sakanashi M, Takasu $\mathrm{N}$ : Vascular lipotoxicity: endothelial dysfunction via fatty-acid-induced reactive oxygen species overproduction in obese Zucker diabetic fatty rats. Endocrinology 2007;148:160-165.

5 Hosick PA, Stec DE: Heme oxygenase, a novel target for the treatment of hypertension and obesity. Am J Physiol Regul Integr Comp Physiol 2012;302:R207-214.

6 Duann P: GEC-targeted HO-1 expression reduces proteinuria in glomerular immune injury. Am J PhysiolRenal Physiol 2009;297:F629.

7 Li M, Kim DH, Tsenovoy PL, Peterson SJ, Rezzani R, Rodella LF, Aronow WS, Ikehara S, Abraham NG: Treatment of obese diabetic mice with a heme oxygenase inducer reduces visceral and subcutaneous adiposity, increases adiponectin levels, and improves insulin sensitivity and glucose tolerance. Diabetes 2008;57:1526-1535.

8 Kim DH, Burgess AP, Li M, Tsenovoy PL, Addabbo F, McClung JA, Puri N, Abraham NG: Heme oxygenasemediated increases in adiponectin decrease fat content and inflammatory cytokines tumor necrosis factor-alpha and interleukin-6 in Zucker rats and reduce adipogenesis in human mesenchymal stem cells. J Pharmacol Exp Ther 2008;325:833-840.

-9 Li M: Treatment of obese diabetic mice with a heme oxygenase inducer reduces visceral and subcutaneous adiposity, increases adiponectin levels, and improves insulin sensitivity and glucose tolerance. Diabetes 2008;57:15261535.

10 Kim DH: Epoxyeicosatrienoic Acid Agonist Regulates Human Mesenchymal Stem Cell-Derived Adipocytes Through Activation of HO-1-pAKT Signaling and a Decrease in PPAR $\gamma$. Stem Cells Dev 2010;19:18631873.

11 Cao J: High fat diet enhances cardiac abnormalities in SHR rats: Protective role of heme oxygenaseadiponectin axis. Diabetol Metab Syndr 2011;3:3737.

12 Iwashima Y, Katsuya T, Ishikawa K, Ouchi N, Ohishi M, Sugimoto K, Fu Y, Motone M, Yamamoto K, Matsuo A, Ohashi K, Kihara S, Funahashi T, Rakugi H, Matsuzawa Y, Ogihara T: Hypoadiponectinemia is an independent risk factor for hypertension. Hypertension 2004;43:1318-1323.

13 Wang B, Yu Y, Han L: Adiponectin improves endothelial dysfunction caused by elevated FFAs levels, partially through cAMP-dependent pathway. Diabetes Res Clin Pract 2012;97:119-124.

14 Cao J, Inoue K, Sodhi K, Puri N, Peterson SJ, Rezzani R, Abraham NG: High-fat diet exacerbates renal dysfunction in SHR: reversal by induction of HO-1-adiponectin axis. Obesity (Silver Spring) 2012;20:945953. 
15 Cheng CF, Lian WS, Chen SH, Lai PF, Li HF, Lan YF, Cheng WT, Lin H: Protective effects of adiponectin against renal ischemia-reperfusion injury via prostacyclin-PPARalpha-heme oxygenase-1 signaling pathway. J Cell Physiol 2012;227:239-249.

16 Nuutila P: Glucose-free fatty acid cycle operates in human heart and skeletal muscle in vivo. J Clin Invest1992;89:1767.

17 Yu YR, Li HL, Zhang XX: [Effects of free fatty acids on nitric oxide synthase activity and mRNA expression in endothelial cell of SD rat aorta]. Sichuan Da Xue Xue Bao Yi Xue Ban 2008;39:193-196.

-18 Arregui B, Lopez B, Garcia SM, Valero F, Navarro C, Fenoy FJ: Acute renal hemodynamic effects of dimanganese decacarbonyl and cobalt protoporphyrin. Kidney Int 2004;65:564-574.

19 Hou N, Han F, Wang M, Huang N, Zhao J, Liu X, Sun X: Perirenal fat associated with microalbuminuria in obese rats. Int Urol Nephrol 2014;46:839-845.

20 Lee RM, Lu C, Su LY, Werstuck G, Gao YJ: Effects of hyperglycemia on the modulation of vascular function by perivascular adipose tissue. J Hypertens 2009;27:118-131.

21 Sun X, Yu Y, Han L: High FFA levels related to microalbuminuria and uncoupling of VEGF-NO axis in obese rats. Int Urol Nephrol 2013;45:1197-1207.

-22 Li R, Wang WQ, Zhang H, Yang X, Fan Q, Christopher TA, Lopez BL, Tao L, Goldstein BJ, Gao F, Ma XL: Adiponectin improves endothelial function in hyperlipidemic rats by reducing oxidative/nitrative stress and differential regulation of eNOS/iNOS activity. Am J Physiol Endocrinol Metab 2007;293:E1703-1708.

-23 Kono T, Saito M, Kinoshita Y, Satoh I, Shinbori C, Satoh K: Real-time monitoring of nitric oxide and blood flow during ischemia-reperfusion in the rat testis. Mol Cell Biochem 2006;286:139-145.

24 Abraham NG, Kushida T, McClung J, Weiss M, Quan S, Lafaro R, Darzynkiewicz Z, Wolin M: Heme oxygenase- 1 attenuates glucose-mediated cell growth arrest and apoptosis in human microvessel endothelial cells. Circ Res 2003;93:507-514.

25 Ohara Y, Peterson TE, Harrison DG: Hypercholesterolemia increases endothelial superoxide anion production. J Clin Invest 1993;91:2546-2551.

26 El MA, de Champlain J: Prevention of hypertension, insulin resistance, and oxidative stress by alpha-lipoic acid. Hypertension 2002;39:303-307.

27 Cooper-DeHoff RM, Pepine CJ: Metabolic syndrome and cardiovascular disease: challenges and opportunities. Clin Cardiol 2007;30:593-597.

28 Abraham NG, Kappas A: Pharmacological and clinical aspects of heme oxygenase. Pharmacol Rev 2008;60:79-127.

29 Abraham NG, Rezzani R, Rodella L, Kruger A, Taller D, Li VG, Goodman AI, Kappas A: Overexpression of human heme oxygenase-1 attenuates endothelial cell sloughing in experimental diabetes. Am J Physiol Heart Circ Physiol 2004;287:H2468-2477.

-30 L'Abbate A, Neglia D, Vecoli C, Novelli M, Ottaviano V, Baldi S, Barsacchi R, Paolicchi A, Masiello P, Drummond GS, McClung JA, Abraham NG: Beneficial effect of heme oxygenase-1 expression on myocardial ischemia-reperfusion involves an increase in adiponectin in mildly diabetic rats. Am J Physiol Heart Circ Physiol 2007;293:H3532-3541.

31 Ouchi N: Association of hypoadiponectinemia with impaired vasoreactivity. Hypertension 2003;42:231234.

32 Ouedraogo R, Gong Y, Berzins B, Wu X, Mahadev K, Hough K, Chan L, Goldstein BJ, Scalia R: Adiponectin deficiency increases leukocyte-endothelium interactions via upregulation of endothelial cell adhesion molecules in vivo. J Clin Invest 2007;117:1718-1726.

-33 Pansuria M, Xi H, Li L, Yang XF, Wang H: Insulin resistance, metabolic stress, and atherosclerosis. Front Biosci (Schol Ed) 2012;4:916-931.

34 Yamauchi T, Kadowaki T: Physiological and pathophysiological roles of adiponectin and adiponectin receptors in the integrated regulation of metabolic and cardiovascular diseases. Int J Obes (Lond) 2008;32:S13-18.

-35 Berg AH, Combs TP, Scherer PE: ACRP30/adiponectin: an adipokine regulating glucose and lipid metabolism. Trends Endocrinol Metab 2002;13:84-89.

-36 Stec DE, Vera T, McLemore GR Jr, Kelsen S, Rimoldi JM, Gadepalli RS, Ryan MJ: Heme oxygenase-1 induction does not improve vascular relaxation in angiotensin II hypertensive mice. Am J Hypertens 2008;21:189193.

-37 Imai T, Morita T, Shindo T, Nagai R, Yazaki Y, Kurihara H, Suematsu M, Katayama S: Vascular smooth muscle cell-directed overexpression of heme oxygenase-1 elevates blood pressure through attenuation of nitric oxide-induced vasodilation in mice. Circ Res 2001;89:55-62. 